\title{
The beneficial effects of berries on cognition, motor behaviour and neuronal function in ageing
}

\author{
Barbara Shukitt-Hale*, Donna F. Bielinski, Francis C. Lau, Lauren M. Willis, Amanda N. Carey and \\ James A. Joseph $\dagger$ \\ USDA-ARS, Human Nutrition Research Center on Aging, Tufts University, Boston, MA 02111, USA
}

(Submitted 18 March 2015 - Final revision received 17July 2015 - Accepted 10 August 2015 - First published online 22 September 2015)

\begin{abstract}
Previously, it has been shown that strawberry (SB) or blueberry (BB) supplementations, when fed to rats from 19 to 21 months of age, reverse age-related decrements in motor and cognitive performance. We have postulated that these effects may be the result of a number of positive benefits of the berry polyphenols, including decreased stress signalling, increased neurogenesis, and increased signals involved in learning and memory. Thus, the present study was carried out to examine these mechanisms in aged animals by administering a control, $2 \%$ SB- or $2 \%$ BB-supplemented diet to aged Fischer 344 rats for 8 weeks to ascertain their effectiveness in reversing age-related deficits in behavioural and neuronal function. The results showed that rats consuming the berry diets exhibited enhanced motor performance and improved cognition, specifically working memory. In addition, the rats supplemented with BB and SB diets showed increased hippocampal neurogenesis and expression of insulin-like growth factor 1, although the improvements in working memory performance could not solely be explained by these increases. The diverse polyphenolics in these berry fruits may have additional mechanisms of action that could account for their relative differences in efficacy.
\end{abstract}

Key words: Strawberries: Blueberries: Spatial memory: Learning: Stress signalling: Neurogenesis

Although the mechanisms involved in motor $^{(1,2)}$ and cognitive ${ }^{(3)}$ behavioural deficits during ageing remain to be discerned, it is clear that oxidative stress (OS) $)^{(3)}$ and inflammation (INF) ${ }^{(4,5)}$ are involved. Increased susceptibility to the long-term effects of OS and inflammatory insults are thought to be contributing factors to the decrements in cognitive and/or motor performance seen in ageing and other neurodegenerative diseases. Deficits in brain functions due to OS may be the result of declines in endogenous antioxidant defence mechanisms ${ }^{(6-11)}$ and increases in the vulnerability of the brain to the deleterious effects of oxidative damage $^{(12)}$. Research also indicates that not only is the CNS particularly vulnerable to OS but that this vulnerability increases during ageing (see Joseph et al. ${ }^{(13,14)}$ for review) and may also enhance central vulnerability to $\operatorname{INF}^{(13,15)}$. With age, there are increases in inflammatory mediators (e.g. cytokines) ${ }^{(16-18)}$, as well as increased mobilisation and infiltration of peripheral inflammatory cells, which have been shown to produce deficits in behaviour similar to those observed during ageing ${ }^{(4)}$. Furthermore, age-related changes in brain vulnerability to OS and INF may be the result of membrane changes and differential receptor sensitivity ${ }^{(19)}$.
The question then becomes how to reduce OS/INF vulnerability to these age-related changes and reverse the aforementioned deficits in motor and cognitive function. In this respect, studies have shown that plants, including fruit- or vegetable-bearing plants, synthesise a vast array of chemical compounds that are not necessarily involved in the plant's metabolism. These 'secondary compounds' instead serve a variety of functions that enhance the plant's survivability, including combating OS and INF. Research suggests that polyphenolic compounds contained in colourful fruits and vegetables exhibit potent antioxidant and antiinflammatory activities that can reduce the age-related sensitivity to OS or $\mathrm{INF}^{(20)}$.

In previous studies, we found that crude blueberry (BB) or strawberry (SB) extracts significantly attenuated age-related motor and cognitive deficits in senescent rodents ${ }^{(21,22)}$, as well as radiation-induced cognitive behavioural decrements in young rats ${ }^{(23)}$. Specifically, young rats given an AIN-93 diet supplemented with SB extract or spinach extract (1-2\% of the diet) for 8 months did not exhibit age-related decrements in cognitive performance or neuronal function that were seen in the unsupplemented controls ${ }^{(22)}$. In a subsequent experiment,

Abbreviations: BB, blueberry; BrdU, bromodeoxyuridine; IGF-1, insulin-like growth factor 1; INF, inflammation; MWM, Morris water maze; OS, oxidative stress; SB, strawberry; TBST, Tris-buffered saline with Tween.

* Corresponding author: B. Shukitt-Hale, fax +1617556 3299, email barbara.shukitthale@ars.usda.gov

$\dagger$ In memory of James A. Joseph, our valued colleague and friend, who passed away while this paper was being written. 
BB or SB supplementation for 8 weeks reversed age-related deficits in neuronal and behavioural (motor and cognitive) function in aged (19 months) Fischer 344 (F344) rats ${ }^{(21)}$. The rodents in all diet groups, but not the control group, showed improved working memory (short-term memory) performance in the Morris water maze (MWM) test ${ }^{(21)}$. Later studies suggested that, in addition to MWM performance, BB supplementation was also effective in reversing cognitive declines in object recognition $^{(24,25)}$.

Subsequent research has suggested that berry fruit polyphenols may possess a multiplicity of actions aside from antioxidant activity. Other possible mechanisms for the berry fruit's positive effects include direct effects on signalling to enhance neuronal communication ${ }^{(26-31)}$, the ability to buffer against excess $\mathrm{Ca}^{(32)}$, increased neurogenesis ${ }^{(27)}$, enhancement of neuroprotective stress shock proteins ${ }^{(33)}$, alteration of inflammatory gene expression and protection against neurodegeneration following excitotoxic stress ${ }^{(34,35)}$, and reduction of stress signals such as $\mathrm{NF}_{\kappa} \mathrm{B}^{(24,28)}$. In addition, the anthocyanins contained in $\mathrm{BB}$ have been shown to enter the brain, and their concentrations were correlated with cognitive performance ${ }^{(36)}$.

The present study was carried out to further assess the behavioural benefits of $\mathrm{BB}$ or $\mathrm{SB}$ in aged rats supplemented with different diets for 8 weeks. In addition, we also assessed insulin-like growth factor 1 (IGF-1) in the hippocampus, a neuronal signal that has been shown to be associated with learning and memory, in the supplemented and control groups. Finally, measurements were made of dentate neurogenesis in control and supplemented animals.

\section{Methods}

\section{Animals}

In all, forty-two 19-month-old male Fischer 344 rats, obtained from the NIA colony (Harlan Sprague Dawley), were individually housed in stainless steel mesh suspended cages, provided food and water ad libitum, and maintained on a $12 \mathrm{~h}$ light-12 h dark cycle. Following a 1-week acclimatisation period to the facility, the rats were weight-matched and then randomly assigned to one of three diet groups until euthanised ( $n$ 14/group): control, $2 \% \mathrm{SB}$ or $2 \% \mathrm{BB}$. Weights were recorded at several time points throughout the study and during behavioural testing. A food intake assessment (over a 96-h period) was made during the 6th week of the experimental feeding. All rats were observed daily for clinical signs of disease. During the course of the study, two rats in the control group, one in the $\mathrm{BB}$ group and two in the $\mathrm{SB}$ group were euthanised because of excessive weight loss. Animals were utilised in compliance with all applicable laws and regulations as well as with principles expressed in the National Institutes of Health, USPHS, Guide for the Care and Use of Laboratory Animals. This study was approved by the Animal Care and Use Committee of our Center.

\section{Diets}

The diets were prepared at Harlan Teklad by adding freezedried fruit powder to the control diet, which was a modification of the NIH-31 diet $(20 \mathrm{~g} / \mathrm{kg}$ diet, $2 \% \mathrm{w} / \mathrm{w})$. The amount of maize in the control diet was adjusted to compensate for the added volume of the fruit powder. The control NIH-31 diet was the same as used in earlier studies in which other fruits, including $\mathrm{BB}$, were found to be beneficial in mitigating brain ageing $(24,26,27,37,38)$. The $\mathrm{BB}$ powder was prepared by homogenising $\mathrm{BB}$, obtained from US Highbush Blueberry Council, with deionised water $(1: 1, \mathrm{w} / \mathrm{v})$ for $3 \mathrm{~min}$ and then centrifuging the recovered homogenate at $27500 \boldsymbol{g}$ for $15 \mathrm{~min}$ at $4^{\circ} \mathrm{C}$. The supernatant was then frozen, crushed and lyophilised. The SB powder was prepared by freeze-drying SB supplied by the California Strawberry Commission; this powder has been previously described ${ }^{(39)}$. The amount of anthocyanins in each diet was determined by liquid chromatography-tandem MS according to the methods of Milbury et $a l .^{(40)}$ (see Table 1).

\section{Behavioural tests}

Psychomotor testing. A battery of age- and diet-sensitive tests of psychomotor behaviour ${ }^{(2,21,37,38,41,42)}$ was administered in a randomised order to the animals during the 8th week of treatment. Each test was performed once, separated by a break between tasks. Rats were tested in a random order, with the restriction that one rat from each diet group be tested in succession. Briefly, the tests included the following: (1) rod walking, which measures psychomotor coordination and the integrity of the vestibular system by requiring the animal to balance on a stationary, horizontal rod; (2) wire suspension, which measures muscle strength and the prehensile reflex, an animal's ability to grasp a horizontal wire with its forepaws and to remain suspended; (3) plank walking, which measures balance and coordination by exposing the rats to horizontal planks of three different widths; (4) inclined screen, which measures muscle tone, strength, stamina and balance by placing the animal on a wire mesh screen that is tilted $60^{\circ}$ to the horizontal plane of the floor; and (5) accelerating rotarod, which measures fine motor coordination, balance and resistance to fatigue by assessing the duration that a rat can remain standing/walking on a

Table 1. Anthocyanin content $(\mathrm{ng} / \mathrm{g})$ of the rat diets as measured by liquid chromatography-tandem $\mathrm{MS}^{(40)}$

\begin{tabular}{|c|c|c|c|}
\hline Anthocyanin & $\begin{array}{l}\text { Control diet } \\
(\mathrm{ng} / \mathrm{g})\end{array}$ & $\begin{array}{l}\text { Blueberry diet } \\
\text { (ng/g) }\end{array}$ & $\begin{array}{c}\text { Strawberry diet } \\
(\mathrm{ng} / \mathrm{g})\end{array}$ \\
\hline Cyanidin-3-galactoside & 0.0 & $4968 \cdot 3$ & 0.0 \\
\hline Cyanidin-3-glucoside & $38 \cdot 7$ & $2081 \cdot 8$ & 4493.9 \\
\hline Cyanidin-3-arabinoside & $21 \cdot 2$ & 601.9 & 0.0 \\
\hline Delphinidin-3-glucoside* & 0.0 & 35.5 & 0.0 \\
\hline Malvidin-3-arabinoside* & 0.0 & 3661.6 & 0.0 \\
\hline Malvidin-3-galactoside* & 0.0 & 9979.5 & 0.0 \\
\hline Malvidin-3-glucoside* & 0.0 & $6985 \cdot 6$ & 0.0 \\
\hline $\begin{array}{l}\text { Pelargonidin-3-(6"- } \\
\text { acetoyl)glucoside* }\end{array}$ & 124.5 & 0.0 & $848 \cdot 8$ \\
\hline Pelargonidin-3-glucoside* & 2149.4 & 0.0 & $121446 \cdot 0$ \\
\hline Peonidin-3-galactoside & 0.0 & $14839 \cdot 3$ & 0.0 \\
\hline Peonidin-3-glucoside & $16 \cdot 5$ & $2933 \cdot 2$ & 0.0 \\
\hline Petunidin-3-glucoside* & 0.0 & 0.0 & $1078 \cdot 7$ \\
\hline
\end{tabular}

* Because of the lack of analytical standards available, analytes were calculated using a molar equivalency to cyanidin-3-glucoside 
rotating, slowly accelerating rod. (For a more detailed description of the tests, see Shukitt-Hale et $a l_{.}^{(42)}$.)

Cognitive testing. The MWM test, an accepted method of testing spatial learning and memory, is an age- ${ }^{(41-43)}$ and dietsensitive ${ }^{(21,37,44)}$ learning paradigm that requires the rat to use spatial learning to find a hidden platform $(10 \mathrm{~cm}$ in diameter) submerged $2 \mathrm{~cm}$ below the surface of the water in a circular pool of water $(134 \mathrm{~cm}$ in diameter $\times 50 \mathrm{~cm}$ in height), maintained at $23^{\circ} \mathrm{C}$, and to remember its location from the previous trial. Accurate navigation is rewarded with escape from the water onto the platform, which the rat effectively locates by using distal cues. The working memory version of the MWM $^{(43,45)}$ was performed daily for 4 consecutive days during the 9th week of treatment, with a morning and an afternoon session, two trials at each session, with a 10-min inter-trial interval between the two trials. Rats were tested in a random manner, with the restriction that one rat from each group be tested in succession. At the beginning of each trial, the rat was gently immersed in water at one of four randomised start locations. Each rat was allowed $120 \mathrm{~s}$ to escape onto the platform; if the rat failed to escape within this time, it was guided to the platform. Once the rat reached the platform, it remained there for $15 \mathrm{~s}$ (trial 1 ; reference memory or acquisition trial). The rat was returned to its home cage between trials (10 min). Trial 2 (the working memory or retrieval trial) used the same platform location and start position as trial 1. Performances were videotaped and analysed with image tracking software (HVS Image), which allows measurements of latency to find the platform (s), path length $(\mathrm{cm})$ and swimming speed $(\mathrm{cm} / \mathrm{s}$; latency/path length). (For a more detailed description of the maze and the paradigm used, see Shukitt-Hale et al. ${ }^{(42)}$.)

\section{Neurogenesis}

At least $10 \mathrm{~d}$ following behavioural testing, the rats were injected with bromodeoxyuridine (BrdU, $50 \mathrm{mg} / \mathrm{kg}$ ) for $3 \mathrm{~d}$. On the 4 th day, one-half of the rats in each diet group ( $n$ 6-7/group) were perfused with PBS and their brains post-fixed in $4 \%$ paraformaldehyde in order to study the proliferation of newly divided cells via immunohistochemistry. The remaining rats ( $n$ 6/group) were perfused $28 \mathrm{~d}$ later, as above, to study cell survival.

For single immunostaining of $\mathrm{BrdU}, 40-\mu \mathrm{m}$ sections were cut with a cryostat, and every tenth section spanning the entire extent of the hippocampus was treated to denature DNA with $2 \mathrm{~N}-\mathrm{HCl}$ and DNase I. Sections (6-8/rat) were incubated with a mouse monoclonal anti-BrdU antibody, followed by incubation with a rat-absorbed biotinylated anti-mouse secondary antibody. Immunoreactivities were visualised by the avidin-biotin-peroxidase procedure using 3,3'-diaminobenzidine (DAB) as chromogen (Vectastain Elite ABC Kit; Vector). For double staining of BrdU and glial fibrillary acidic protein (GFAP), sections were treated and stained for BrdU as described above, except that $\mathrm{Ni}$ was used in conjunction with DAB to produce a black colouration. After BrdU staining, sections were blocked with normal horse serum, incubated with a polyclonal rabbit anti-GFAP primary antibody and followed by incubation with a biotinylated anti-rabbit secondary antibody. GFAP-positive cells were detected with the ABC Kit using DAB alone to obtain brown-stained cells. BrdU-positive cells were counted by two independent raters. Estimates were made of the total number of positive cells located throughout the entire extent of the granular cell layer (GCL), including the subgranular zone (as defined by a two-cellbody-wide zone at the edge of the GCL) and the hilus, as well as these regions separately, to examine migration pattern changes. The estimated total number of BrdU-positive cells for each hippocampus was calculated by first taking the mean count between two raters for each sectioned dentate gyrus area, then averaging the means of six sections counted per hippocampus, and multiplying this average for 6 sections by 60 (60 being the total number of sections across the analysed portion of hippocampus for each animal).

\section{Western immunoblotting: insulin-like growth factor 1}

For analysis of IGF-1 by western immunoblotting, sample preparation was done according to a slightly modified procedure of Williams et al. ${ }^{(30)}$; specifically, hippocampal brain regions were homogenised on ice with a Teflon-pestle motorised handheld homogeniser in a buffer containing Tris $(50 \mathrm{~mm})$, Triton $\mathrm{X}-100(0 \cdot 1 \%), \mathrm{NaCl}(150 \mathrm{~mm})$ and ethylene glycol tetraacetic acid/EDTA (2 mm), pH 7.4, with mammalian protease inhibitor cocktail (1:100 dilution), sodium pyrophosphate (1 mm), phenylmethanesulfonylfluoride $(10 \mu \mathrm{g} / \mathrm{ml})$, sodium vanadate ( $1 \mathrm{~mm}$ ) and sodium fluoride $(50 \mathrm{~mm})$. Homogenates were left on ice for $45 \mathrm{~min}$ before centrifugation at $1000 \mathrm{~g}$ for $5 \mathrm{~min}$ at $4^{\circ} \mathrm{C}$ to remove unbroken cell debris and nuclei. Protein concentration in the supernatants was determined by the Dc Protein Assay (Bio-Rad). Gel samples were boiled for $5 \mathrm{~min}$ in Laemmli sample buffer with a final concentration of $2.5 \% 2$ mercaptoethanol. Samples were stored at $-80^{\circ} \mathrm{C}$ until analysis.

Western immunoblotting procedures were conducted as per Ferrer et $a l .{ }^{(46)}$ and Mendelson et al. ${ }^{(47)}$. Briefly, $20 \mu \mathrm{g}$ of protein samples and pooled protein standard were run on a $12.5 \%$ SDS-PAGE gel. The gel was soaked in protein transfer buffer (200 mm-glycine, 25 mm-Tris base, $20 \%$ methanol) for $10 \mathrm{~min}$, and then proteins were electrophoretically transferred to a nitrocellulose membrane. Blots were blocked with TBST (Trisbuffered saline with Tween; $140 \mathrm{~mm}-\mathrm{NaCl}, 20 \mathrm{~mm}$-Tris, $0.05 \%$ Tween-20, pH 7.5) with $4 \%$ non-fat dried milk and incubated in the IGF-1 antibody (Millipore) in TBST without milk, followed by washing with TBST and incubation with the appropriate horseradish peroxidase-conjugated secondary antibody in TBST without milk. After a final washing in TBST, the signal was detected using ECL Plus Reagents. The immunoreactive bands, corresponding to the molecular weight of the protein of interest, were visualised with a digital charge-coupled device camera (Hamamatsu Photonics) attached to a BioImaging System (EC 3 Darkroom; UVP), and the optical densities were quantified with LabWorks Imaging Acquisition and Analysis software (version 4.5; UVP). The pooled protein standard was used to normalise the intensities of the antibody-specific bands, and the signals were expressed as percentage of optical density of the standard for comparison of signal across the blots. 


\section{Statistical analyses}

For each measure, between-subjects ANOVA models comparing the diet groups were performed using Systat (SPSS Inc.) to test for statistical significance at the $P<0.05$ level. Days or trials, when appropriate, were included in the model as a within-subjects variable. Post hoc comparisons, to determine differences between the diet groups, were performed using Fisher's least significant difference post hoc analysis. To analyse working memory, separate $t$ tests were conducted for each group between the trial 1 and trial 2 latencies. Correlations between behaviour and brain measures were carried out using Pearson's $r$ correlation.

\section{Results}

There were no differences in weight $(P>0.05$; average weight: control group $=428.41 \quad(\operatorname{sem} 10 \cdot 27) ; \quad$ BB group $=431.71$ $(\operatorname{sem} 7.02) ; \quad$ SB group $=428.67(\operatorname{sem} 7.43))$ or food intake $(P>0.05$; average food intake: control group $=21.74$ ( (sem 0.88); $\mathrm{BB}$ group $=22.20 \quad(\operatorname{sem} 0.71) ; \mathrm{SB}$ group $=19 \cdot 88(\operatorname{sem} 1.16))$ between the diet groups during the study.

\section{Psychomotor tests}

Differences in psychomotor performance were seen among the diet groups (Fig. 1). Specifically, latency to fall in the rod walking test (mean values with their standard errors) was higher in the BB group compared with the SB group $(P<0.05)$; however, neither diet group was different from the control group (group effect: $F_{2,34}=4.30 ; P<0.05$; Fig. 1(a)). Rotarod performance was improved by the $\mathrm{BB}$ diet compared with control $(P<0.05)$, whereas the SB group latency was not different from either the control or the BB group (group effect: $F_{2,34}=2 \cdot 35 ; P=0.10 ;$ Fig. $\left.1(\mathrm{~b})\right)$. On the large plank walk test, the $\mathrm{SB}$ group had a longer latency to fall compared with the $\mathrm{BB}$ group $(P<0.05)$, whereas neither diet group was different from the control group (group effect: $F_{2,34}=4.52 ; P<0 \cdot 05$; Fig. $1(\mathrm{c}$ )). However, the $\mathrm{BB}$ group also made significantly more $180^{\circ}$ turns on the large plank compared with the SB or control group $(P<0.05)$, which might have contributed to the shorter latency to fall (group effect: $F_{2,34}=3.31 ; P<0.05$; data not shown). There were no additional differences between the groups on any of the other motor tests.

\section{Cognitive tests}

When examining cognitive performance, the berry fruit diet groups showed improved performance compared with the control diet group (Fig. 2). There were no overall group differences in MWM latency or distance to the platform in trial 1 or trial 2 performance. However, separate $t$ tests were performed between the two trial latencies or distances for each group for days 3 and 4 (the days when performance relies more on memory than on learning) to determine whether the different diet groups significantly improved their performance from trial 1 to trial 2 , an indication of working memory. We found that rats in both the $\mathrm{BB}(t(12)=3.21 ; P=0.007)$ and $\mathrm{SB}$ $(t(11)=2 \cdot 11 ; P=0.05)$ groups showed a significant reduction in latency to find the platform between trial 1 and trial 2; that is trial 2 latencies were significantly less than trial 1 latencies, showing that these rats demonstrated one-trial learning, even with the 10-min retention interval (Fig. 2(a)). This one-trial learning was not found in the control group $(t(11)=1 \cdot 04$; $P=0.32)$. In addition, both the $\mathrm{BB}(t(12)=3.51 ; P=0.004)$ and SB $(t(11)=3 \cdot 14 ; \quad P=0.009)$ diet groups showed significant decreases in distance to find the platform between trial 1 and trial 2; that is, trial 2 distances were significantly less than trial 1 distances (Fig. 2(b)), whereas this difference was not different in the control group $(t(11)=1.44 ; P=0 \cdot 178)$. Therefore, rats consuming the berry fruit diets showed reversal of the deleterious effects of ageing on cognitive performance, particularly on working memory. These differences were not due to swim speed as there were no differences between the groups in this parameter on days 3 and 4 .

(A)

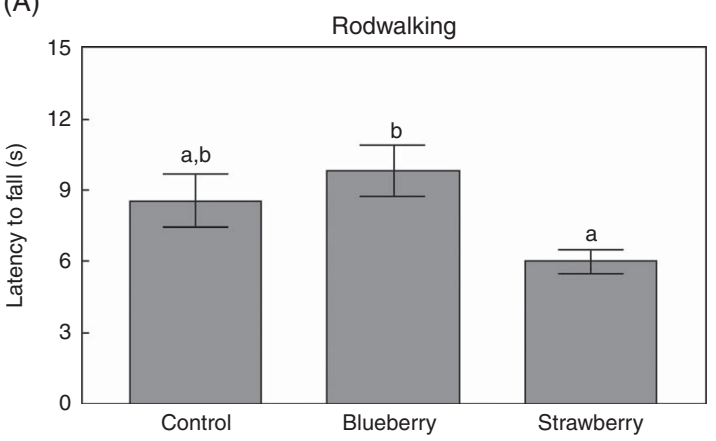

(B)

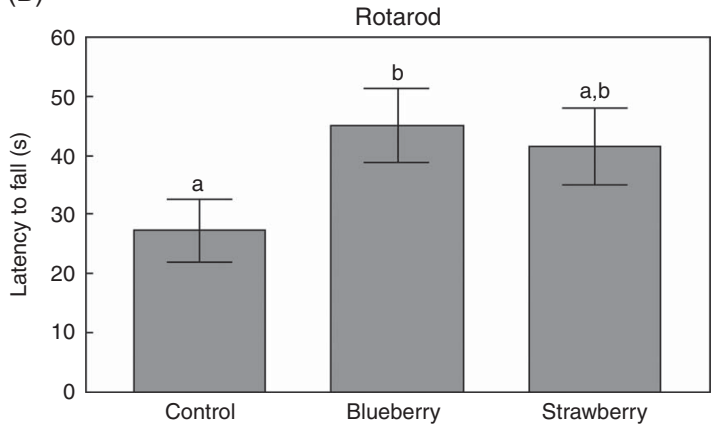

(C)

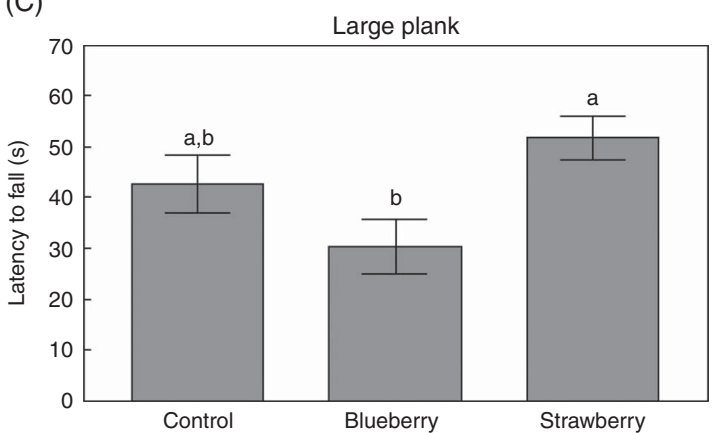

Fig. 1. Latency to fall in the rod walking (A), rotarod (B) and large plank (C) tests for the control, $2 \%$ blueberry and $2 \%$ strawberry diet groups. Values are means, with their standard errors represented by vertical bars. ${ }^{a, b}$ Mean values with unlike letters were significantly different $(P<0.05$; Fisher's least significant difference). 
(A)

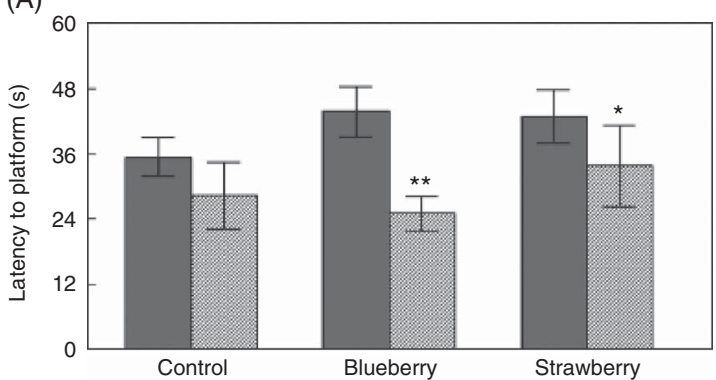

(B)

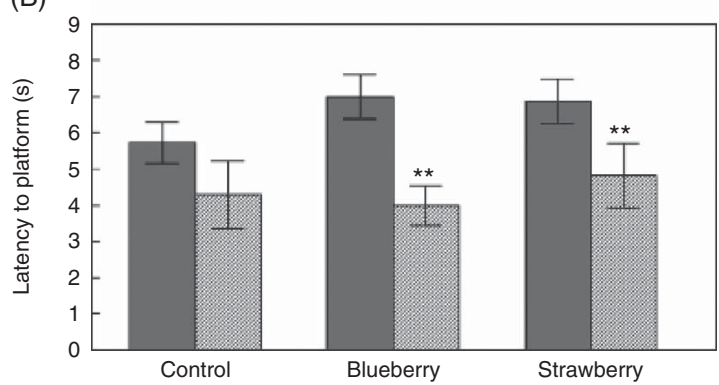

Fig. 2. Morris water maze performance assessed as latency in seconds (A) and distance in metres (B) to find the hidden platform over days 3 and 4 of testing for animals in the control, $2 \%$ blueberry and $2 \%$ strawberry diet groups. Values are means, with their standard errors represented by vertical bars. Statistical difference in performance (i.e. an improvement) between $\square$, trial 1 and 㖘, trial $2\left({ }^{*} P<0.05,{ }^{* *} P<0.01\right)$, indicating improved working memory.

\section{Neurogenesis}

Differences were seen in cell survival among the diet groups (group effect: $F_{2,12}=2.95 ; P=0.09$; Fig. $3(\mathrm{a})$ ), with rats in the SB group showing a significant increase in the number of cells surviving in the dentate gyrus of the hippocampus compared with the control diet group $(P<0 \cdot 05)$. However, even though rats in the SB group also showed higher numbers of proliferating precursor cells (Fig. 3(b)), these differences did not reach significance $(P>0.05)$. In both measures, rats in the $\mathrm{BB}$ diet group showed numbers that fell between those of the $\mathrm{SB}$ and control rats, and were not different from either group (Fig. 3(a) and (b)). The BrdU-positive cells did not co-localise with the GFAP-positive cells (data not shown).

Correlations between neurogenesis and cognitive performance revealed that, as the number of proliferating cells increased, the mean difference in latency between trial 1 and trial 2 on days 3-4 also increased ( $r 0.414 ; P=0 \cdot 087$; Fig. 4$)$. The difference score is a measure of working memory, or short-term memory, in that the higher the difference score, the more improvement from trial 1 to trial 2, as the score implies that the rats are able to find the platform more quickly on trial 2 by remembering where it was on trial 1 , after the 10 min delay. Interestingly, when examined separately by diet group, only the BB group showed a significant correlation between proliferation and working memory performance $(r 0.816 ; P<0.05)$; neither the control group $(r 0.290 ; P>0.05)$ nor the SB group ( $r$ 0.040; $P>0.05$ ) yielded significance. In contrast, cognitive performance did not significantly correlate with surviving cells,
(A)

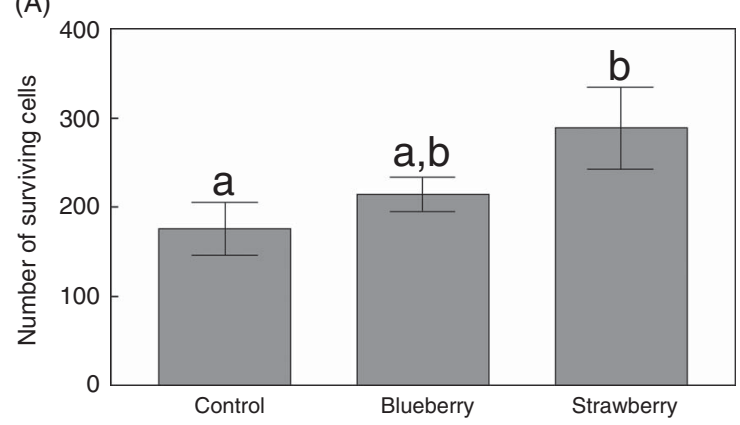

(B)

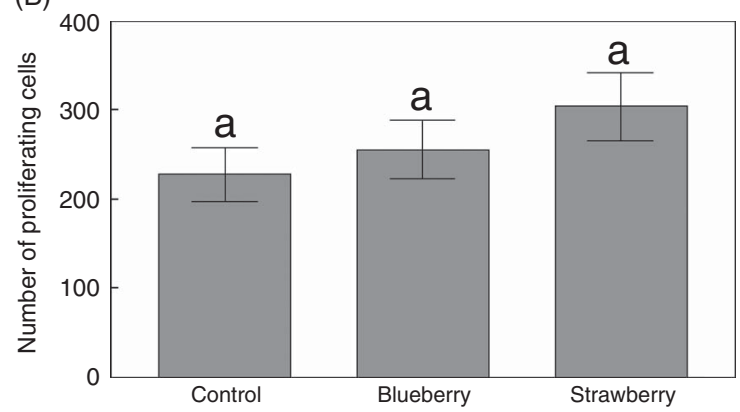

Fig. 3. The number of surviving (A) and proliferating (B) precursor cells (measured as BrdU-positive cells) in the dentate gyrus of the hippocampus of rats in the control, $2 \%$ blueberry and $2 \%$ strawberry diet groups. Values are means, with their standard errors represented by vertical bars. ${ }^{\mathrm{a}, \mathrm{b}}$ Mean values with unlike letters were significantly different $(P<0.05$; Fisher's least significant difference).

and none of the motor tests showed a positive correlation with neurogenesis.

\section{Western immunoblotting}

IGF-1 levels were increased by both berry diets compared with control $(P<0.05)$, but levels in the SB group were also significantly higher $(P<0 \cdot 05)$ than those in the BB group (group effect: $F_{2,12}=33.70 ; \quad P<0.05 ;$ Fig. 5). Correlations between IGF-1 and cognitive performance revealed no overall relationship between IGF-1 levels and the mean difference in latency between trial 1 and trial 2 on days 3-4 ( $r-0.028 ; P>0.05)$. However, when examined separately by diet group, only the BB group showed a positive correlation between IGF-1 levels and working memory performance (meaning that, as IGF-1 levels increased, the difference score for latency from trial 1 to trial 2 increased, showing improved learning), although this value did not reach statistical significance $(r 0 \cdot 772 ; P=0 \cdot 126)$. Correlation values for both the control group $(r-0.884$; $P<0.05)$ and the SB group $(r-0.357 ; P>0.05)$ were negative, although only the value for the control group was statistically significant, showing that higher IGF-1 levels were associated with poorer working memory performance in this group.

\section{Discussion}

Overall, results from this study showed that consuming berrysupplemented diets led to enhanced motor performance, improved cognition, specifically working memory, increased 


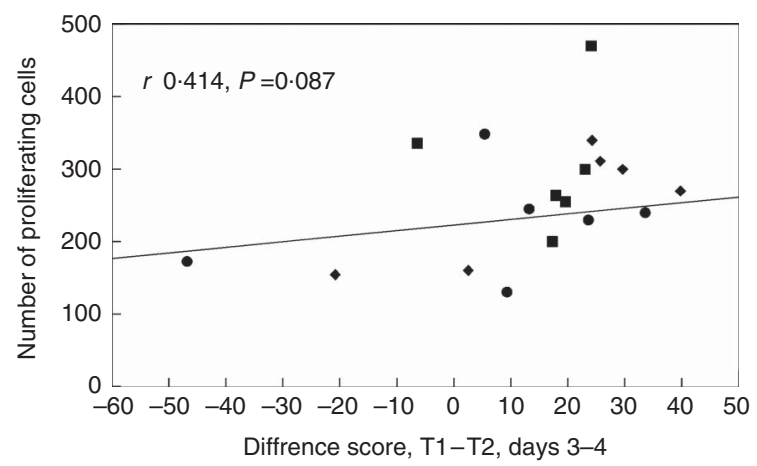

Fig. 4. Correlations between neurogenesis (number of proliferating cells) and cognitive behaviour (difference score of trial 1 (T1) and trial 2 (T2) in the Morris water maze on days $3-4$ ) of rats in the control, $2 \%$ blueberry (BB) and $2 \%$ strawberry (SB) diet groups. Control: $r 0.290, P>0.05$; $\$$, BB: $r 0.816$, $P<0.05 ;$, SB: $r 0.040, P>0.05$.

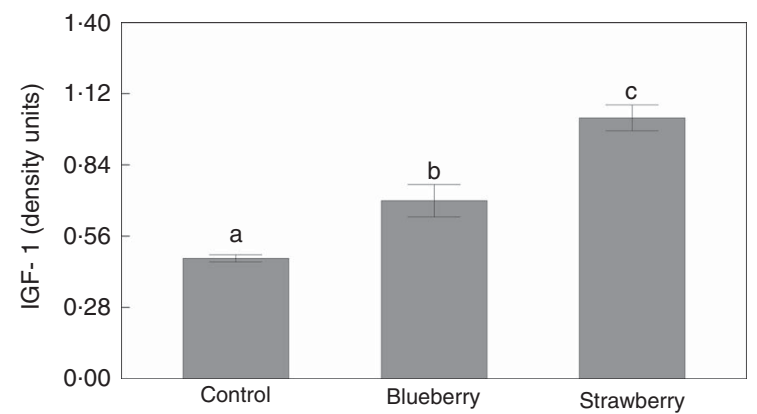

Fig. 5. Insulin-like growth factor 1 (IGF-1) levels in the hippocampus of rats in the control, $2 \%$ blueberry and $2 \%$ strawberry diet groups. Quantitative measurements were based on the western blots and represented as mean band density. ${ }^{a, b, c}$ Mean values with unlike letters were significantly different $(P<0.05$; Fisher's least significant difference).

neurogenesis and increased IGF-1 signalling. However, these results also indicate that the diverse polyphenolics in the different berry fruits might be acting differentially to produce their positive effects. For example, on the motor tests, the BB diet group was better on the rod walk, which measures psychomotor coordination and the integrity of the vestibular system, whereas the SB group was better on the plank walk, which assesses more general balance and coordination. Both supplemented groups showed positive effects on the rotarod test, which is a measure of fine coordination, balance and resistance to fatigue. Conversely, both diets showed positive effects on the cognitive tests. However, the improvements in working memory performance could not solely be explained by the increases in neurogenesis and neuronal signalling. For example, only the behaviour of the BB group was significantly correlated with the number of proliferating cells in the dentate gyrus, even though overall the SB group had the highest number of proliferating cells and a significantly greater number of surviving cells compared with any other group. Similarly, only the behaviour in the $\mathrm{BB}$ group showed a positive relationship with IGF-1 levels, even though the SB-fed group had the highest IGF-1 levels.

Because both berry fruit diets did not produce equivalent results, the types and relative amounts of polyphenols in the different berries could account for their relative differences in efficacy. Therefore, we measured the anthocyanin content of the diets as we feel these polyphenols are the most relevant and the most distinct among these two fruits. The control diet had trace amounts of a few anthocyanins. However, the BB diet showed higher levels of four different anthocyanins, specifically cyanidin, delphinidin, malvidin and peonidin, compared with the SB diet, which had more pelargonidin and petunidin than the $\mathrm{BB}$ diet. In fact, the only anthocyanin that the two berry diets had in common was cyanidin-3-glucoside. Whereas the BB diet had smaller amounts of more anthocyanins, the anthocyanin content of the SB diet was driven by the large quantity of pelargonidin-3-glucoside. We have shown previously that anthocyanins contained in $\mathrm{BB}$, specifically the four seen in the rat diet in this study, cross the blood-brain barrier in glycosylated form and localise in the brain, and the total number of anthocyanin compounds in the cortex and hippocampus correlates with MWM performance ${ }^{(36)}$. Future studies should measure polyphenols and their metabolites in the serum and brain of rats, to determine which specific anthocyanins contribute to the improvements in behaviour.

These results agree with an earlier study in our laboratory ${ }^{(27)}$ that found that aged rats fed a $2 \%$ BB-supplemented diet had significant increases in hippocampal proliferation of neuronal precursor cells compared with non-supplemented aged rats, and these increases correlated with reductions in the number of memory errors in a radial arm water maze. Furthermore, this study found that BB-fed rats had increases in the neuroprotective trophic factor IGF-1 that were associated with significant decreases in memory errors ${ }^{(27)}$. Another study found that rats fed a $2 \% \mathrm{BB}$ diet prior to central administration of the neurotoxin kainic acid showed increased hippocampal mRNA expression of IGF-1 compared with control-fed rats ${ }^{(34)}$. In the present study, spatial working memory performance in the BB group was also associated with increases in hippocampal plasticity parameters of neurogenesis and IGF-1 levels. Interestingly, these associations were not seen in the SB group. It remains to be determined whether changes in other markers would correlate better with the cognitive improvements in the SB group or whether another measure of cognition would correlate better with these markers.

Given the well-documented link between hippocampal neurogenesis, cognitive performance and age, the fact that an association was found between the proliferation of neuronal precursor cells and spatial memory performance in our animals suggests that berry-mediated increases in neurogenesis may play a key role in the reported improvements in cognition in such aged animals. However, despite this association between proliferation and cognition, neither berry diet group showed a significantly higher number of proliferating cells compared with the control group, possibly because of the small number of rats in each group measured for neurogenesis (6/group). It is also surprising that, even though the berry diets increased the survival of hippocampal neurons, spatial working memory in the MWM did not correlate with this parameter. Perhaps proliferation itself is more important in improving spatial memory and learning in the BB-supplemented animals. Alternatively, it may be that diet-induced changes in survival 
take a longer time to develop, and the length of the feeding prior to behavioural testing was not a long enough time to capture this change. It is also possible that $\mathrm{BB}$ may be more effective at stimulating neuronal differentiation and in improving neuroplasticity compared with SB; future studies should examine these measures.

Another animal study showed that supplementation with a BB diet ( $2 \%$ of the diet for 12 weeks) improved spatial working memory in aged rats in a cross maze, and that, as in the present study, these performance improvements were associated with attenuated levels of a variety of signalling molecules involved in stress, survival and neural plasticity in the hippocampus ${ }^{(30)}$. Memory performance correlated with the activation of cyclic AMP-response element-binding protein and increases in brain-derived neurotrophic factor in the hippocampus, which were linked to increases in the phosphorylation state of extracellular signal-related kinase and changes in the activation state of mammalian target of rapamycin. Interestingly, one key pathway that the IGF-1 receptor signals through is regulated by phosphatidylinositol-3 kinase and its downstream partner mTOR $^{(48)}$

The current study highlights the need to study the effects of berry fruits on human health and, to date, only a few studies have investigated changes in cognition with age. Most notably, in one study, older adults with mild cognitive impairment consuming BB juice (6-9 ml/kg per d) for 12 weeks showed improved word list recall and increased paired associate learning, relative to both baseline and placebo controls ${ }^{(49)}$. Recent clinical research has also linked changes in walking speed and gait variability to declines in cognitive ability during 'normal' ageing ${ }^{(50,51)}$. On the basis of these findings and existing evidence for the protective effects of berry fruits on motor ability in rodent models, from this and previous studies, it can be stated that future clinical research should incorporate measures of cognition paired with measures of motor control, balance and mobility to determine whether berry supplementation can enhance motor control in older, nonpathological, adults.

Taken together, these preclinical findings suggest that dietary supplementation with berry fruits has the potential for a multiplicity of effects, in addition to their antioxidant and anti-inflammatory properties, including the activation of a variety of signalling pathways that result in neuroprotection, neurogenesis and, ultimately, spared cognitive and motor behaviour. Individual polyphenols in the berry fruits might exert their effects through different and/or independent mechanisms. Future studies should assess which specific polyphenolic compounds and/or metabolites enter the serum, and from there into which areas of the brain in animals, to ascertain the extent to which regional localisation and bioavailability of the $\mathrm{BB}$ and $\mathrm{SB}$ polyphenols contribute to their mechanism(s) of action.

\section{Acknowledgements}

B. S.-H. thanks Jason Walsh, $\mathrm{PhD}$, and Oliver Chen, $\mathrm{PhD}$, of the Antioxidants Research Lab at the HNRCA at Tufts University for their help in identifying the anthocyanins in the rat chow.
This research was supported by USDA intramural funds and agreements between the USDA and the Wild Blueberry Association of North America (WBANA), the US Highbush Blueberry Council (USHBC) and the California Strawberry Commission.

A. N. C and B. S.-H. ran the behavioural tests and analysed the data; D. F. B. ran the western blots; L. M. W. and F. C. L. performed the neurogenesis assays and analysed the results; J. A. J. and B. S.-H. designed the experiments and prepared the manuscript; and B. S.-H. had primary responsibility for final content. All authors have read and approved the final manuscript.

There are no conflicts of interest.

\section{References}

1. Kluger A, Gianutsos JG, Golomb J, et al. (1997) Patterns of motor impairment in normal aging, mild cognitive decline, and early Alzheimer's disease. J Gerontol 52, 28-39.

2. Joseph JA, Bartus RT, Clody D, et al. (1983) Psychomotor performance in the senescent rodent: reduction of deficits via striatal dopamine receptor up-regulation. Neurobiol Aging $\mathbf{4}$, 313-319.

3. Shukitt-Hale B (1999) The effects of aging and oxidative stress on psychomotor and cognitive behavior. Age (Omaha) 22, 9-17.

4. Hauss-Wegrzyniak B, Vannucchi MG \& Wenk GL (2000) Behavioral and ultrastructural changes induced by chronic neuroinflammation in young rats. Brain Res 859, 157-166.

5. Hauss-Wegrzyniak B, Vraniak P \& Wenk GL (1999) The effects of a novel NSAID on chronic neuroinflammation are age dependent. Neurobiol Aging 20, 305-313.

6. Halliwell B (1994) Free radicals and antioxidants: a personal view. Nutr Rev 52, 253-265.

7. Harman D (1981) The aging process. Proc Natl Acad Sci US A 78, 7124-7128.

8. Yu BP (1994) Cellular defenses against damage from reactive oxygen species [published erratum appears in Physiol Rev 1995 75(1):preceding 1]. Physiol Rev 74, 139-162.

9. Olanow CW (1992) An introduction to the free radical hypothesis in Parkinson's disease. Ann Neurol 32, S2-S9.

10. Carney JM, Smith CD, Carney AN, et al. (1994) Aging- and oxygen-induced modifications in brain biochemistry and behavior. Ann N Y Acad Sci 738, 44-53.

11. Gilissen EP, Jacobs RE \& Allman JM (1999) Magnetic resonance microscopy of iron in the basal forebrain cholinergic structures of the aged mouse lemur. J Neurol Sci 168, 21-27.

12. Olanow CW (1993) A radical hypothesis for neurodegeneration. Trends Neurosci 16, 439-444.

13. Joseph JA, Denisova N, Fisher D, et al. (1998) Age-related neurodegeneration and oxidative stress: putative nutritional intervention. Neurol Clin 16, 747-755.

14. Joseph JA, Denisova N, Fisher D, et al. (1998) Membrane and receptor modifications of oxidative stress vulnerability in aging. Nutritional considerations. Ann N Y Acad Sci 854, 268-276.

15. Joseph J, Shukitt-Hale B, Denisova NA, et al. (2001) Copernicus revisited: amyloid beta in Alzheimer's disease. Neurobiol Aging 22, 131-146.

16. Rozovsky I, Finch CE \& Morgan TE (1998) Age-related activation of microglia and astrocytes: in vitro studies show. Neurobiol Aging 19, 97-103.

17. McGeer PL \& McGeer EG (1995) The inflammatory response system of the brain: implications for therapy of Alzheimer and other neurodegenerative diseases. Brain Res Brain Res Rev 21, 195-218. 
18. Volpato S, Guralnik JM, Ferrucci L, et al. (2001) Cardiovascular disease, interleukin-6, and risk of mortality in older women: the women's health and aging study. Circulation 103, 947-953.

19. Joseph JAD, NA, Youdim KA, Bielinski D, et al. (2001) Neuronal environment and age-related neurodegenerative disease: nutritional modification. In Annual Review of Gerontology and Geriatrics, Focus on Modern Topics in the Biology of Aging, vol. 21, pp. 195-235 [VJ Cristofalo and A Richard, editor]. New York, NY: Springer Publishing Co.

20. Stevenson DE \& Hurst RD (2007) Polyphenolic phytochemicals - just antioxidants or much more? Cell Mol Life Sci 64, 2900-2916.

21. Joseph JA, Shukitt-Hale B, Denisova NA, et al. (1999) Reversals of age-related declines in neuronal signal transduction, cognitive, and motor behavioral deficits with blueberry, spinach, or strawberry dietary supplementation. J Neurosci 19, 8114-8121.

22. Joseph JA, Shukitt-Hale B, Denisova NA, et al. (1998) Long-term dietary strawberry, spinach, or vitamin E supplementation retards the onset of age-related neuronal signal-transduction and cognitive behavioral deficits. J Neurosci 18, 8047-8055.

23. Shukitt-Hale B, Carey AN, Jenkins D, et al. (2007) Beneficial effects of fruit extracts on neuronal function and behavior in a rodent model of accelerated aging. Neurobiol Aging 28, 1187-1194.

24. Goyarzu P, Malin DH, Lau FC, et al. (2004) Blueberry supplemented diet: effects on object recognition memory and nuclear factor-kappa B levels in aged rats. Nutr Neurosci $\mathbf{7}$, $75-83$.

25. Malin DH, Lee DR, Goyarzu P, et al. (2010) Short-term blueberry-enriched diet prevents and reverses object recognition memory loss in aging rats. Nutrition 27, 338-342.

26. Joseph JA, Arendash G, Gordon M, et al. (2003) Blueberry supplementation enhances signaling and prevents behavioral deficits in an Alzheimer disease model. Nutr Neurosci 6 , 153-162.

27. Casadesus G, Shukitt-Hale B, Stellwagen HM, et al. (2004) Modulation of hippocampal plasticity and cognitive behavior by short-term blueberry supplementation in aged rats. Nutr Neurosci 7, 309-316.

28. Joseph JA, Bielinski DF \& Fisher DR (2010) Blueberry treatment antagonizes C-2 ceramide-induced stress signaling in muscarinic receptor-transfected COS-7 cells. J Agri Food Chem 58, 3380-3392.

29. Joseph JA, Shukitt-Hale B, Brewer GJ, et al. (2010) Differential protection among fractionated blueberry polyphenolic families against DA-, Abeta(42)- and LPS-induced decrements in Ca (2+) buffering in primary hippocampal cells. J Agri Food Chem 58, 8196-8204.

30. Williams CM, El Mohsen MA, Vauzour D, et al. (2008) Blueberry-induced changes in spatial working memory correlate with changes in hippocampal CREB phosphorylation and brain-derived neurotrophic factor (BDNF) levels. Free Radic Biol Med 45, 295-305.

31. Rendeiro C, Vauzour D, Kean RJ, et al. (2012) Blueberry supplementation induces spatial memory improvements and region-specific regulation of hippocampal BDNF mRNA expression in young rats. Psychopharmacology (Berl) 223 319-330.

32. Joseph JA, Fisher DR \& Carey AN (2004) Fruit extracts antagonize Abeta- or DA-induced deficits in Ca2+ flux in M1-transfected COS-7 cells. I Alzheimers Dis 6, 403-411; discussion 443-409.

33. Galli RL, Bielinski DF, Szprengiel A, et al. (2006) Blueberry supplemented diet reverses age-related decline in hippocampal HSP70 neuroprotection. Neurobiol Aging 27, 344-350.
34. Shukitt-Hale B, Lau FC, Carey AN, et al. (2008) Blueberry polyphenols attenuate kainic acid-induced decrements in cognition and alter inflammatory gene expression in rat hippocampus. Nutr Neurosci 11, 172-182.

35. Duffy KB, Spangler EL, Devan BD, et al. (2008) A blueberryenriched diet provides cellular protection against oxidative stress and reduces a kainate-induced learning impairment in rats. Neurobiol Aging 29, 1680-1689.

36. Andres-Lacueva C, Shukitt-Hale B, Galli RL, et al. (2005) Anthocyanins in aged blueberry-fed rats are found centrally and may enhance memory. Nutr Neurosci 8, 111-120.

37. Youdim KA, Shukitt-Hale B, Martin A, et al. (2000) Short-term dietary supplementation of blueberry polyphenolics: beneficial effects on aging brain performance and peripheral tissue function. Nutr Neurosci 3, 383-397.

38. Shukitt-Hale B, Galli R, Meterko V, et al. (2005) Dietary supplementation with fruit polyphenolics ameliorates age-related deficits in behavior and neuronal markers of inflammation and oxidative stress. Age(Dordr) 27, 49-57.

39. Seeram NP, Lee R, Scheuller HS, et al. (2006) Identification of phenolic compounds in strawberries by liquid chromatography electrospray ionization mass spectroscopy. Food Chem 97, 1-11.

40. Milbury PE, Vita JA \& Blumberg JB (2010) Anthocyanins are bioavailable in humans following an acute dose of cranberry juice. J Nutr 140, 1099-1104.

41. Ingram DK, Jucker M \& Spangler EL. (1994) Behavioral manifestations of aging. In Pathobiology of the Aging Rat, pp. 149-170 [U Mohr, DL Cungworth and CC Capen, editors]. Washington, DC: ILSI.

42. Shukitt-Hale B, Mouzakis G \& Joseph JA (1998) Psychomotor and spatial memory performance in aging male Fischer 344 rats. Exp Gerontol 33, 615-624.

43. Brandeis R, Brandys Y \& Yehuda S (1989) The use of the Morris water maze in the study of memory and learning. Intern J Neurosci 48, 29-69.

44. Shukitt-Hale B, Carey A, Simon LE, et al. (2004) Fruit polyphenols prevent inflammatory mediated decrements in cognition. Soc Neurosci Abs 30, 565.565.

45. Morris R (1984) Development of a water-maze procedure for studying spatial learning in the rat. $J$ NeurosciMethods $\mathbf{1 1}$, 47-60.

46. Ferrer I, Blanco R, Carmona M, et al. (2002) Active, phosphorylation-dependent MAP kinases, MAPK/ERK, SAPK/ JNK and p38, and specific transcription factor substrates are differentially expressed following systemic administration of kainic acid to the adult rat. Acta Neuropathol 103, 391-407.

47. Mendelson KG, Contois LR, Tevosian SG, et al. (1996) Independent regulation of $\mathrm{JNK} / \mathrm{p} 38$ mitogen-activated protein kinases by metabolic oxidative stress in the liver. Proc Natl Acad Sci U S A 93, 12908-12913.

48. O'Neill C, Kiely AP, Coakley MF, et al. (2012) Insulin and IGF-1 signalling: longevity, protein homoeostasis and Alzheimer's disease. Biochem Soc Trans 40, 721-727.

49. Krikorian R, Shidler MD, Nash TA, et al. (2010) Blueberry supplementation improves memory in older adults. J Agri Food Chem 58, 3996-4000.

50. Watson NL, Rosano C, Boudreau RM, et al. (2010) Executive function, memory, and gait speed decline in well-functioning older adults. J Gerontol A Biol Sci Med Sci 65, 1093-1100.

51. Soumare A, Tavernier B, Alperovitch A, et al. (2009) A crosssectional and longitudinal study of the relationship between walking speed and cognitive function in community-dwelling elderly people. I Gerontol A Biol Sci Med Sci 64, 1058-1065. 